\title{
An Intelligent Information Sharing Strategy \\ within a Swarm for Unconstrained and Constrained Optimization Problems
}

\author{
TAPABRATA RAY AND K. M. LIEW \\ Center for Advanced Numerical Engineering Simulations \\ School of Mechanical and Production Engineering \\ Nanyang Technological University \\ Singapore 639798 \\ Email: mtray@ntu.edu.sg;mkmliew@ntu.edu.sg
}

PANKAJ SAINI

Lucent Technologies Singapore Pte Ltd

Software Products Group

6 Battery Road,\#28 01/02 Singapore 049909

Email: psaini@lucent.com

\begin{abstract}
In this paper we present a new multilevel information sharing strategy within a swarm to handle single objective, constrained and unconstrained optimization problems. A swarm is a collection of individuals having a common goal to reach the best value (minimum or maximum) of a function. Among the individuals in a swarm, there are some better performers (leaders) those that set the direction of search for the rest of the individuals. An individual that is not in the better performer list (BPL) improves its performance by deriving information from its closest neighbor in BPL. In an unconstrained problem, the objective values are the performance measures used to generate the BPL while a multilevel Pareto ranking scheme is implemented to generate the BPL for constrained problems. The information sharing strategy also ensures that all the individuals in the swarm are unique as in a real swarm, where at a given time instant two individuals cannot share the same location. The uniqueness among the individuals result in a set of near optimal individuals at the final stage that is useful for sensitivity analysis. The benefits of the information sharing strategy
\end{abstract}


within a swarm are illustrated by solving two unconstrained problems with multiple equal and unequal optimum, a constrained optimization problem dealing with a test function and a well studied welded beam design problem.

Keywords: Pareto, Constrained Optimization, and Swarm Strategy.

\section{Introduction}

Swarm strategies are fairly new methods that have received considerable attention over recent years as optimization methods for complex functions. Kennedy and Eberhart (1995) initially proposed the swarm strategy for optimization. Unlike conventional evolutionary methods, the swarm strategy is based on simulation of social behavior where each individual in a swarm adjusts its flying according to its own flying experience and campanions' flying experience. The key to the success of such a strategy in solving an optimization problem lies with the mechanism of information sharing across individuals to collectively attain a common goal. After the initial concept was proposed, there had been comparative studies between swarm and other evolutionary strategies by Eberhart and Shi (1998) and Angeline (1998).

It is well known that the presence of constraints significantly affects the performance of all optimization algorithms including evolutionary search methods. There has been a number of approaches to handle constraints in the domain of evolutionary methods including rejection of infeasible individuals, penalty functions and their variants, repair methods, use of decoders, separate treatment of constraints and objectives and hybrid methods incorporating knowledge of constraint satisfaction. Michalewicz (1995) provides a comprehensive review on constraint handling methods. All the methods have limited success as they are problem dependent and require a number of additional inputs. Penalty functions using static, dynamic or adaptive concepts have been developed over the years. All of them suffer from common problems of aggregation and scaling. Repair methods are based on additional function evaluations, while the decoders and special operators or constraint satisfaction methods are problem specific and cannot be used to model a generic constraint. 
Separate treatment of constraints and objectives is an interesting concept that eliminates the problem of scaling and aggregation.

Constraint handling using a Pareto ranking scheme is a relatively new concept having its origin in multiobjective optimization. Fonseca and Flemming (1995) proposed the Multiple Objective Genetic Algorithm (MOGA), a Pareto ranking scheme to handle multiple objectives while the Nondominated Sorting Genetic Algorithm (NSGA) was introduced by Srinivas and Deb (1994) for multiobjective problems. Ray et al. (2000) introduced a multiobjective evolutionary algorithm that is based on Pareto ranking in both the objective and constraint domain suitable for a wide variety of single or multiobjective constraint problems.

For a swarm strategy to be efficient for constrained problems there is additional information about the constraint satisfaction that needs to be meaningfully shared among the individuals. The information sharing strategy proposed in this paper relies on Pareto methods to handle constraints. The strategy also ensures that distinct points are maintained throughout the search, as such points are useful for sensitivity analysis. The proposed multilevel information sharing strategy is described in detail in the next section separately for unconstrained and constrained problems.

\section{Mathematical Formulation}

A general constrained optimization problem (in minimization sense) is presented as:

$$
\begin{array}{cc}
\text { Minimize } & f(\mathbf{x}) \\
\text { subject to } & g_{i}(\mathbf{x}) \geq a_{i}, i=\mathbf{1}, \mathbf{2}, \ldots, q \\
& h_{j}(\mathbf{x})=b_{j}, j=\mathbf{1}, \mathbf{2}, \ldots, r
\end{array}
$$

where there are $q$ inequality and $r$ equality constraints and $\mathbf{x}=\left[\begin{array}{llll}x_{1} & x_{2} & \ldots & x_{n}\end{array}\right]$ is the vector of $n$ design variables. 
It is work, the equality constraints (with a tolerance $\delta$ ) are transformed to a set of inequalities and a unified formulation is used for all the constraints:

$h_{j}(\mathbf{x}) \leq b_{j}+\delta$ which is same as $-h_{j}(\mathbf{x}) \geq-b_{j}-\delta$ and $h_{j}(\mathbf{x}) \geq b_{j}-\delta$

Thus $r$ equality constraints will give rise to $2 r$ inequalities, and the total number of inequalities for the problem is denoted by $s$, where $s=q+2 r$. For each individual, c denotes the constraint satisfaction vector given by $\mathbf{c}=\left[\begin{array}{lllll}c_{1} & c_{2} & \ldots & c_{s}\end{array}\right]$ where

$$
c_{i}= \begin{cases}0 & \text { if } i^{\text {th }} \text { constraint satisfied , } i=1,2, \ldots, s \\ a_{i}-g_{i}(\mathbf{x}) & \text { if } i^{\text {th }} \text { constraint violated , } i=1,2, \ldots, q \\ b_{i}-\delta-h_{i}(\mathbf{x}) & \text { if } i^{\text {th }} \text { constraint violated , } i=q+1, q+2, \ldots, q+r \\ -b_{i}-\delta+h_{i}(\mathbf{x}) \text { if } i^{\text {th }} \text { constraint violated , } i=q+r+1, q+r+2, \ldots, s\end{cases}
$$

For the above $c_{i}$ 's, $c_{i}=0$ indicates the $i^{\text {th }}$ constraint is satisfied, whereas $c_{i}>0$ indicates the violation of the constraint.

The CONSTRAINT matrix for a swarm of $M$ individuals assumes the form

$$
\text { CONSTRAINT }=\left[\begin{array}{cccc}
c_{11} & c_{12} & \cdots & c_{1 s} \\
c_{21} & c_{22} & \cdots & c_{2 s} \\
\vdots & \vdots & \ddots & \vdots \\
c_{M 1} & c_{M 2} & & c_{M s}
\end{array}\right]
$$

In a swarm of $M$ individuals, all nondominated individuals based on the constraint matrix are assigned a rank of 1 . The rank 1 individuals are removed from the swarm and the new set of nondominated individuals is assigned a rank of 2 . The process is continued until every individual in the swarm is assigned a rank. Rank=1 in the constraint matrix indicate that the individual is nondominated. It can be observed from the constraint matrix that when all the individuals in the swarm are infeasible, the nondominated ones will have a rank of 1 . Whenever there is one or more feasible individual in the swarm, the feasible solutions will take over the as rank 1 individuals.

The initial swarm consists of a collection of random individuals. Over time, the individuals communicate with their neighboring better performers and derive 
information from them to look for optima in smaller groups. The search strategy is schematically shown in Figure 1.

\section{Figure 1. Interaction among Individuals and their Group Leader}

Having described briefly the general formulation of the constraint matrix and the concept of Pareto ranking, the pseudo code of the algorithm is presented.

\subsection{Algorithm}

Initialize $M$ unique individuals in the Swarm;

Unconstrained Problem Strategy

Do \{

Compute Objective Values of each Individual in the Swarm;

Compute Average Objective Value for the Swarm;

For (each Individual) \{

If (Objective Value of an Individual $\leq$ Average Objective Value for the Swarm)

Assign Individual to Better Performer List (BPL);

\}

For (each Individual not in BPL) \{

Do \{

Select a Leader from BPL to derive information;

Acquire information from the Leader and move to a new point in the search space;

\} while (all individuals are not unique)

\}

\} while (termination condition $=$ false $)$ 


\section{Constrained Problem Strategy}

Do \{

Compute Objective values for each Individual in the Swarm;

Compute Constraint values for each Individual in the Swarm;

Use Non Dominated Sorting to Rank Individuals based on Constraint Matrix;

Assign all Individuals with Constraint Rank = 1 to BPL;

If (size of BPL > M/2) \{

Compute Average Objective Value for the Individuals in the BPL;

Shrink the BPL to contain Individuals with Objective value $\leq$ Average

Objective Value;

\}

For (each Individual not in BPL) \{

Do \{

Select a Leader from the BPL to derive information;

Acquire information from the Leader and move to a new point in the search space;

\} while (all individuals are not unique)

\}

\} while (termination condition $=$ false)

For a constrained problem, individuals with a constraint rank $=1$ (nondominated based on constraint matrix) are the best performers based on constraint satisfaction. When there are no feasible individuals in the swarm, the BPL consists of all nondominated individuals based on the constraint matrix. When there is one or more feasible individual, the BPL will consist of all feasible individuals as they will have a constraint rank $=1$. The size of BPL is expected to grow, as more and more individuals become feasible. If the size of this list grows more than half the swarm size (i.e. half of the swarm consists of feasible individuals), individuals are allowed to be members of BPL only if they are better than average performers based on objective values. This process ensures that there is a pressure maintained among the feasible BPL members to improve their objective performance to remain as BPL members. 


\subsection{Selection of Leader}

An individual identifies its closest BPL member in the variable space as its leader to derive information. The process is similar as in a real swarm where an individual adjusts its flying based on information derived from its better performing neighbors.

\subsection{Acquiring Information through Generational Operator}

A simple generational operator is used to acquire information from a leader. The operator can result in a variable value even if it does not exist in either the individual or its leader, which is useful to avoid premature convergence. The probability of a variable value generated between an individual and its leader is $50 \%$. The probability of a variable value generated between the lowerbound of the variable and the minimum among the individual and its leader or between the upperbound of the variable and the maximum among the individual and its leader is $25 \%$ each.

\section{Examples}

The performance of the algorithm is illustrated by the following examples. The first two examples are typically interesting as they illustrate the behavior of swarm and highlight its capability in finding multiple optima simultaneously. The next constrained problem and the engineering design example illustrate the applicability of the algorithm to solve a wide variety of design optimization problems.

\subsection{Equal Optimum Problem}

This example is obtained from Goldberg and Richardson (1987). It is a single variable problem that has five equal optima. The minimization of the function is carried out using the proposed strategy.

Minimize $f(x)=-1.0 * \sin ^{6}(5.1 \pi x+0.5)$ 
where $0.0 \leq x \leq 1.0$. The initial swarm is presented in Figure 2 while the top $50 \%$ of the final swarm is presented in Figure 3. The swarm consists of 50 individuals flying for 100 time steps. Figure 4 shows a distribution of near optimal individuals forming smaller subgroups of 2 to 7 individuals near each optimum with functional values varying between -0.9991 and -1.000 .

It can be observed from Figure 4 that the individuals arrived in groups of 2 to 7 to all the five global optima.

\section{Figure 2. Initial Swarm \\ Figure 3. Final Top 50\% of Swarm Figure 4. Final Top 50\% of Swarm}

\subsection{Unequal Optimum Problem}

This example is also taken from Goldberg and Richardson (1987). It is a single variable problem that has five unequal optimums. The minimization of the function is carried out using the proposed strategy with a swarm size of 50 and for 100 time steps.

$$
\text { Minimize } f(x)=-1.0 * \sin ^{6}(5.1 \pi x+0.5) \exp \left(\frac{-4 \ln (2)(x-0.0667)^{2}}{0.8^{2}}\right)
$$

where $0.0 \leq x \leq 1.0$. The initial swarm is presented in Figure 5 . The top $50 \%$ of the final swarm is presented in Figure 6 that shows the distribution of near optimal points. It can be observed that all the BPL members have reached the actual optimum.

Figure 5. Initial Swarm 


\section{Figure 6. Final Top 50\% of Swarm}

\subsection{Constrained Problem}

This is a constrained single objective optimization problem from Koziel and Michalewicz (1999) that has two variables, a single cubic objective function and is subjected to two nonlinear inequalities. The ratio of feasible points to sampled

$$
\begin{array}{lc}
\text { Minimize } & f(x)=\left(x_{1}-10\right)^{3}+\left(x_{2}-20\right)^{3} \\
\text { Subject to } \quad\left(x_{1}-5\right)^{2}+\left(x_{2}-5\right)^{2}-100 \geq 0 \\
-\left(x_{1}-6\right)^{2}-\left(x_{2}-5\right)^{2}+82.81 \geq 0 \\
13 \leq x_{1} \leq 100, \quad 0 \leq x_{2} \leq 100 .
\end{array}
$$

number of points for a 1,000,000 point random sampling is reported to be 0.000066 .

The optimum individual is [14.095, 0.84296] with an objective function value of 6961.81381. The first two constraints are active at the optimum.

Table 1 provides the results obtained using a swarm size of 200 flying for 100 time steps and also with a swarm size of 300 flying for 100 time steps. Five successive trials have been conducted to compute the best, worst and the average values of the objective using the present algorithm. With a swarm size of 200 and for 100 time steps, the worst, average and the best values are [-6494.0585, 6687.3350, -6916.2939] while for a swarm size of 300 flying for 100 time steps the worst, average and the best values are [-6729.7983, -6837.4347, -6883.7124]. It can be clearly observed from Table 2 that our algorithm reports consistent values with much less evaluations when compared with Koziel and Michalewicz (1999).

\section{Table 1. Results of the Constrained Optimization Problem Table 2. Comparison of Results}




\subsection{Design of a Welded Beam}

This example deals with a welded beam that is to be designed for minimum cost subject to constraints on shear stress, bending stress, buckling load and the end deflection. The four design variables $h, l, t$ and $b$ are shown in Figure 7. The mathematical formulation can be obtained from Rao (1996). This problem has been solved by Deb (1991) using a simple genetic algorithm with a traditional penalty function, and by Ragsdell and Phillips (1976) using geometric programming. Siddall (1972) solved the problem with a suite of optimization techniques: ADRANS - Gall's adaptive random search with penalty function; APPROX - Griffith and Stewart's successive linear approximation; DAVID Davidon-Fletcher-Powell with penalty function; MEMGRD - Miele's memory gradient with penalty function; SIMPLEX - Simplex method with penalty function and RANDOM - Richardson's random method. Coello (1999) used a genetic algorithm with self-adaptive penalty functions to handle the constraints for the same problem.

\section{Figure 7. Welded Beam Design}

The problem involes 7 constraints and the variable bounds are as follows : $0.1 \leq h \leq 2.0,0.1 \leq l \leq 10.0,0.1 \leq t \leq 10.0$, and $0.1 \leq b \leq 2.0$. The results of the welded beam design are presented in Table 3 and Table 4 . The best solution is $[0.167880,4.645894,8.928757,0.219162]$ with a function value of 1.9000 obtained using 38,744 function evaluations.

\section{Table 3. Results of Welded Beam Design}

Table 4 provides a comparison of results with other various methods.

\section{Table 4. Comparison of Results}


It is worth mentioning that Coello (1999) required 900,000 function evaluations while Deb (1991) required 1350 function evaluations.

\section{Summary and Conclusions}

The key to the success of the proposed swarm strategy lies with the intelligent multilevel information sharing mechanism. Pareto ranking and the generation of unique individuals are two computationally expensive operations employed within the algorithm. If the computation of the objective is itself expensive as in the case of most engineering design applications, it is meaningful to make use of all computed information to better guide the search. In addition to that, the use of Pareto ranking and multilayer information sharing for constrained problems eliminate the need of scaling and aggregation affecting common constraint handling methods. The generation of unique individuals on the other hand allows the algorithm to maintain near optimal points that are useful for sensitivity analysis. A simple generational operator has been implemented within this algorithm and other generational operators may well be used instead.

Results of the equal optimum problem clearly illustrate the capability of the algorithm to reach multiple optimal optimums simultaneously. From the unequal optimum example, it is evident that the algorithm is capable to direct individuals to cluster around the global optimum instead of local optima.

For the constrained problem, the use of Pareto ranking with intelligent constraint information sharing leads to a faster convergence as can be seen by the drastic reduction in the number of function evaluations. For the welded beam example, our swarm strategy reached a solution within $8.67 \%$ of the best reported solution by Coello (1999) using a mere 38,744 function evaluations whereas 900,000 function evaluations were required by Coello (1999).

The constrained and unconstrained examples clearly highlight the benefits that can be derived using the new information sharing strategy within the swarm to solve a wide variety of optimization problems from the domain of engineering design. We have listed the average function values and the average function 
evaluations required for various swarm sizes and time steps. Both increasing the swarm size and running the algorithm for a larger number of time steps generally improve the quality of the solution. A comprehensive study is necessary to conclusively identify the effects of swarm size and time steps on the quality of solution obtained.

\section{References}

1. Angeline, P. (1998). Evolutionary Optimization versus Particle Swarm Optimization: Philosophy and Performance Differences, Proceedings of the Seventh Annual Conference on Evolutionary Programming, March 1998.

2. Coello, C.A.C. (1999). Self-adaptive penalties for GA based optimization, Proceedings of the Congress on Evolutionary Computation, Vol. 1, pp. 573-580.

3. Deb, K. (1991). Optimal design of a welded beam via genetic algorithms, AIAA Journal, 29(11), pp. 2013-2015.

4. Eberhart, R. and Shi, Y. (1998). Comparison between genetic algorithms and particle swarm optimization, Proceedings of the Seventh Annual Conference on Evolutionary Programming, Springer Verlag, pp. 611-618.

5. Fonseca, C.M. and Flemming, P.J. (1995). An overview of evolutionary algorithms in multiobjective optimization, Evolutionary Computation, 3(1), pp. 1-16.

6. Goldberg, D.E. and Richardson, J. (1987). Genetic algorithms with sharing for multimodal function optimization, Proceedings of the First International Conference on Genetic Algorithms and Their Applications, pp. 41-49.

7. Kennedy, J., and Eberhart, R. C. (1995). Particle swarm optimization. Proceedings of the 1995 IEEE International Conference on Neural Networks (Perth, Australia), IEEE Service Center, Piscataway, NJ, IV, pp. 1942-1948.

8. Koziel, S. and Michalewicz, Z. (1999). Evolutionary algorithms, homomorphous mappings, and constrained parameter optimization, Evolutionary Computation, 7(1), pp.19-44.

9. Michalewicz, Z. (1995). A survey of constraint handling techniques in evolutionary computation methods, Proceedings of the Fourth Annual Conference on Evolutionary Programming, MIT Press, Cambridge, MA, pp. 135-155.

10. Ragsdell, K.M. and Phillips, D.T. (1976). Optimal design of a class of welded structures using geometric programming, ASME Journal of Engineering for Industry, 98(3), Series B, pp. 1021-1025.

11. Rao, S.S. (1996). Engineering Optimization, John Wiley and Sons, Third Edition.

12. Ray, T., Tai, K. and Seow, K.C. (2000) An evolutionary algorithm for constrained optimization, Proceedings of the Genetic and Evolutionary Computation Conference (GECCO-2000), pp. 771-777.

13. Siddall, J.N. (1972). Analytical decision-making in engineering design, Prentice Hall.

14. Srinivas, N. and Deb, K. (1994). Multiobjective optimization using nondominated sorting in genetic algorithms, Evolutionary Computation, 2(3), pp. 221-248. 


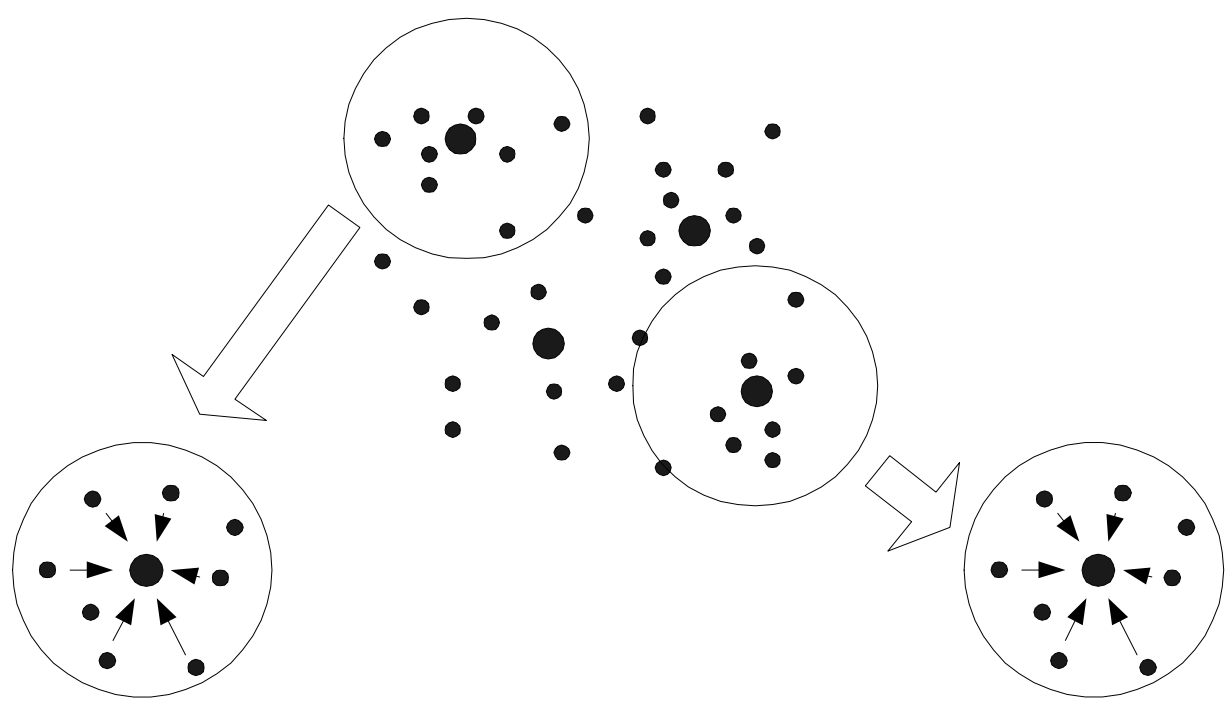

Figure 1. Interaction among Individuals and their Group Leader

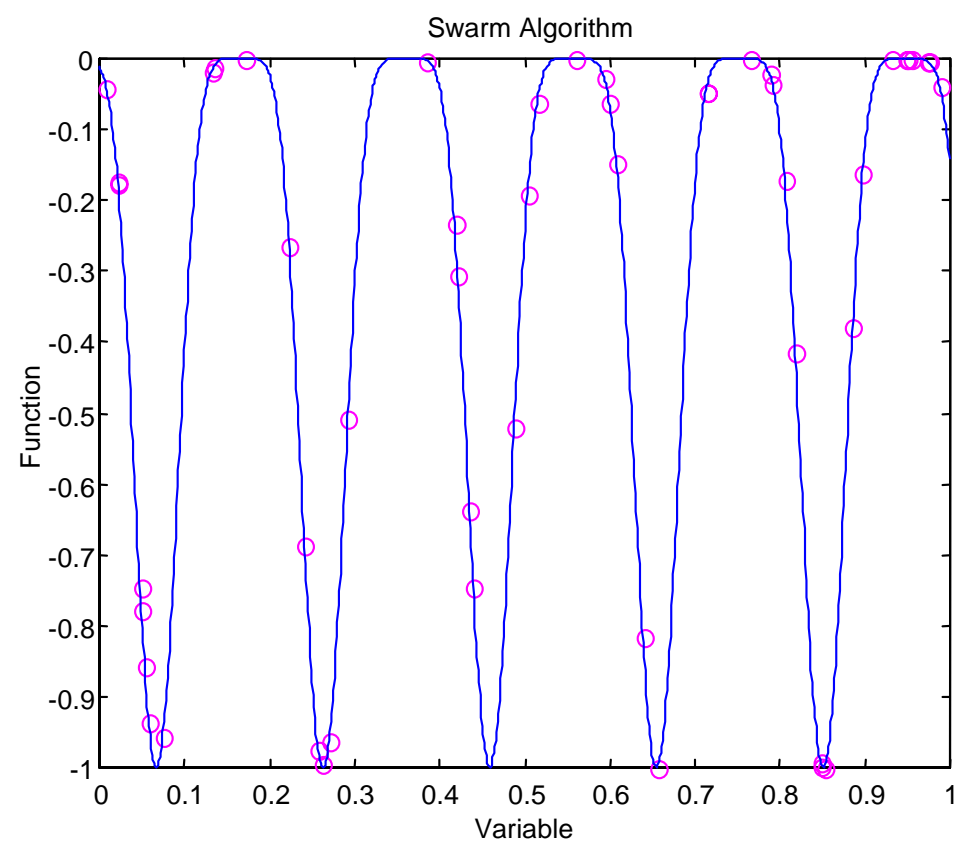

Figure 2: Initial Swarm 


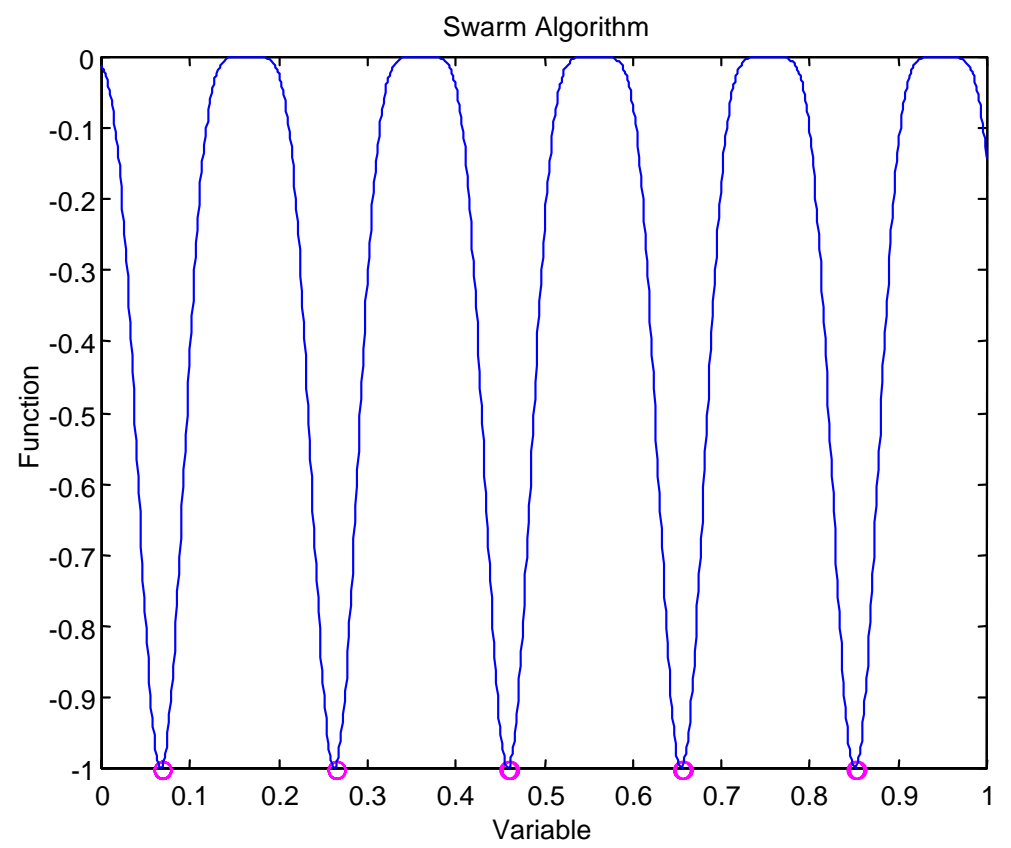

Figure 3: Final Top 50\% of Swarm

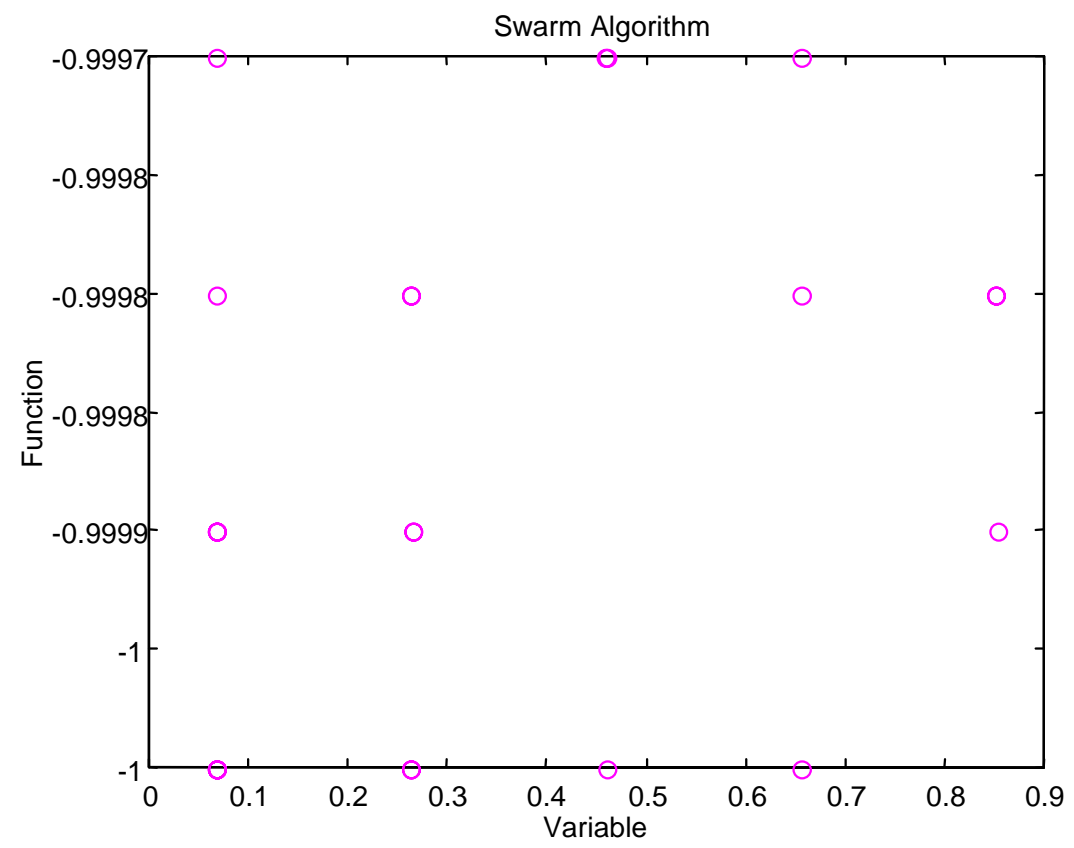

Figure 4: Final Top 50\% of Swarm 


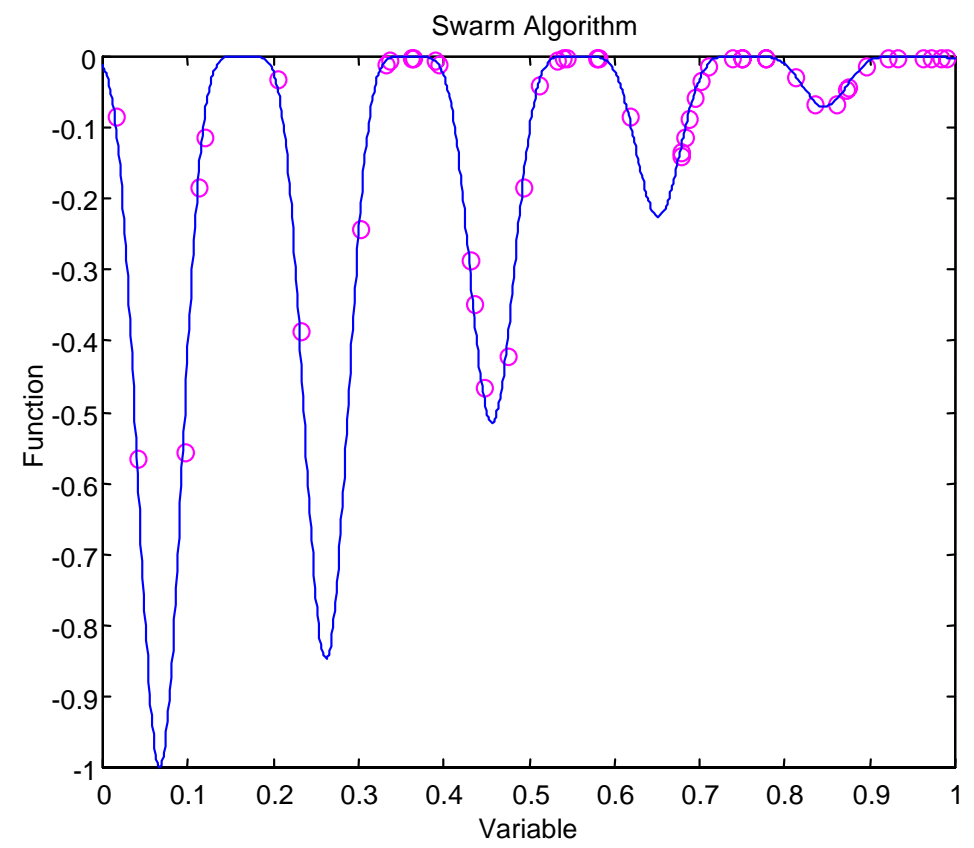

Figure 5: Initial Swarm

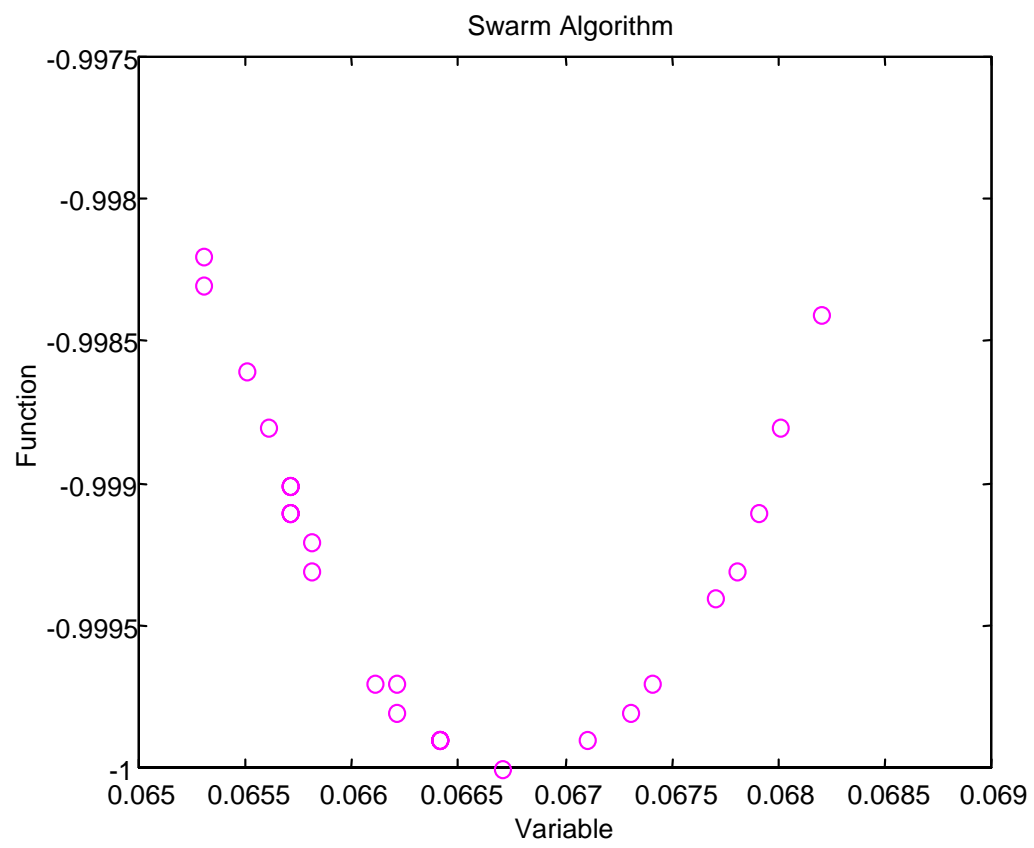

Figure 6: Final Top 50\% of Swarm 


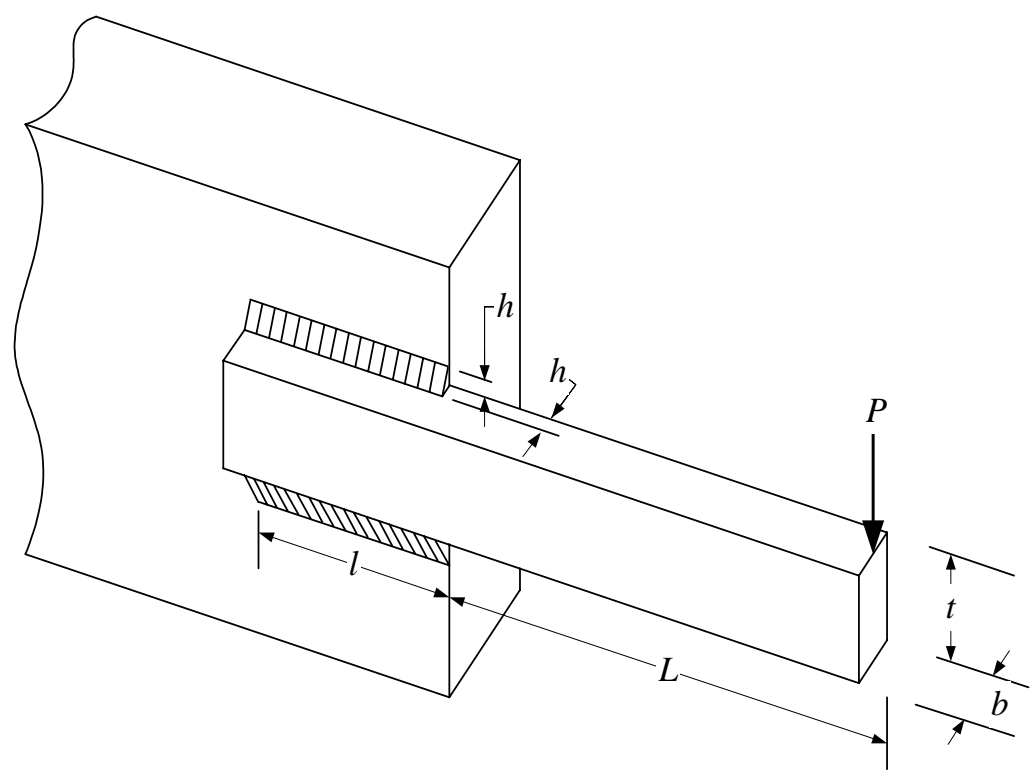

Figure 7: Welded Beam Design 
Table 1: Results of the Constrained Optimization Problem

\begin{tabular}{|l|l|l|}
\hline $\begin{array}{l}\text { Swarm Size }: 200 \\
\text { Time Steps }: 100\end{array}$ & Function Evals. & Obj. Fn \\
\hline $1^{\text {st }}$ Flight & 14,258 & -6668.0268 \\
\hline $2^{\text {nd }}$ Flight & 14,200 & -6596.9409 \\
\hline $3^{\text {rd }}$ Flight & 14,188 & -6494.0585 \\
\hline $4^{\text {th }}$ Flight & 14,355 & -6761.3549 \\
\hline $5^{\text {th }}$ Flight & 16,990 & -6916.2939 \\
\hline & & \\
\hline $\begin{array}{l}\text { Swarm Size }: 300 \\
\text { Time Steps }: 100\end{array}$ & & \\
\hline $1^{\text {st }}$ Flight & 23,318 & \\
\hline $2^{\text {nd }}$ Flight & 23,270 & -6872.4970 \\
\hline $3^{\text {rd }}$ Flight & 22,075 & -6883.7124 \\
\hline $4^{\text {th }}$ Flight & 21,687 & -6729.7983 \\
\hline $5^{\text {th }}$ Flight & 22,721 & -6828.2749 \\
\hline
\end{tabular}

Table 2: Comparison of Results

\begin{tabular}{|l|r|l|}
\hline & $\begin{array}{c}\text { Number of Function } \\
\text { Evaluations }\end{array}$ & Function Value \\
\hline $\begin{array}{l}\text { Koziel and } \\
\text { Michalewicz } \\
(1999)\end{array}$ & 350,000 & -4236.7 (worst) \\
\hline $\begin{array}{l}\text { Koziel and } \\
\text { Michalewicz } \\
(1999)\end{array}$ & -6901.5 (best) \\
\hline $\begin{array}{l}\text { Ray et al. } \\
(2000)\end{array}$ & $1,400,000$ & -5473.9 (worst) \\
& & -6342.6 (avg.) \\
\hline Present & 38,234 & -6525.1 (best) \\
\hline
\end{tabular}


Table 3: Results of Welded Beam Design

\begin{tabular}{|l|l|l|}
\hline $\begin{array}{l}\text { Swarm Size }: 100 \\
\text { Time Steps }: 100\end{array}$ & Function Evals. & Obj. Fn \\
\hline $1^{\text {st }}$ Flight & 6,675 & 2.4298 \\
\hline $2^{\text {nd }}$ Flight & 6,628 & 2.3400 \\
\hline $3^{\text {rd }}$ Flight & 6,521 & 2.3871 \\
\hline $4^{\text {th }}$ Flight & 6,624 & 2.0764 \\
\hline $5^{\text {th }}$ Flight & 6,706 & 2.0983 \\
\hline Average & 6,631 & 2.2583 \\
\hline $\begin{array}{l}\text { Swarm Size }: 200 \\
\text { Time Steps }: 100\end{array}$ & & \\
\hline $1^{\text {st }}$ Flight & 13,167 & \\
\hline $2^{\text {nd }}$ Flight & 13,235 & 2.0123 \\
\hline $3^{\text {rd }}$ Flight & 13,128 & 2.0156 \\
\hline $4^{\text {th }}$ Flight & 13,205 & 1.9088 \\
\hline $5^{\text {th }}$ Flight & 13,226 & 1.9199 \\
\hline Average & 13,192 & 1.9441 \\
\hline $\begin{array}{l}\text { Swarm Size }: 300 \\
\text { Time Steps }: 100\end{array}$ & & 1.9061 \\
\hline $1^{\text {st }}$ Flight & 19,853 & \\
\hline $2^{\text {nd }}$ Flight & 19,582 & 2.0757 \\
\hline $3^{\text {rd }}$ Flight & 19,722 & 2.0379 \\
\hline $4^{\text {th }}$ Flight & 19,603 & 1.9138 \\
\hline $5^{\text {th }}$ Flight & 19,861 & 1.9269 \\
\hline Average & 19,778 & 1.9621 \\
\hline & & 1.9833 \\
\hline
\end{tabular}


Table 4: Results of Welded Beam Design

\begin{tabular}{|l|l|l|}
\hline $\begin{array}{l}\text { Swarm Size : } 100 \\
\text { Time Steps }: 200\end{array}$ & Function Evals. & Obj. Fn \\
\hline $1^{\text {st }}$ Flight & 12,975 & 1.9672 \\
\hline $2^{\text {nd }}$ Flight & 12,989 & 2.0183 \\
\hline $3^{\text {rd }}$ Flight & 12,996 & 2.1588 \\
\hline $4^{\text {th }}$ Flight & 12,947 & 1.9235 \\
\hline $5^{\text {th }}$ Flight & 12,811 & 2.0613 \\
\hline Average & 12,944 & 2.0258 \\
\hline $\begin{array}{l}\text { Swarm Size }: 200 \\
\text { Time Steps }: 200\end{array}$ & & \\
\hline $1^{\text {st }}$ Flight & 25,866 & \\
\hline $2^{\text {nd }}$ Flight & 25,711 & 2.0236 \\
\hline $3^{\text {rd }}$ Flight & 25,820 & 1.9424 \\
\hline $4^{\text {th }}$ Flight & 25,760 & 1.9974 \\
\hline $5^{\text {th }}$ Flight & 25,853 & 1.9282 \\
\hline Average & 25,802 & 1.9476 \\
\hline $\begin{array}{l}\text { Swarm Size }: 300 \\
\text { Time Steps }: 200\end{array}$ & & 1.9678 \\
\hline $1^{\text {st }}$ Flight & 38,744 & \\
\hline $2^{\text {nd }}$ Flight & 38,981 & 1.9000 \\
\hline $3^{\text {rd }}$ Flight & 38,843 & 1.9388 \\
\hline $4^{\text {th }}$ Flight & 38,745 & 1.9114 \\
\hline $5^{\text {th }}$ Flight & 38,487 & 2.0245 \\
\hline Average & 38,760 & 1.9486 \\
\hline & & 1.9446 \\
\hline
\end{tabular}

Table 5: Comparison of Results

\begin{tabular}{|l|c|c|c|c|c|}
\hline & $h$ & $l$ & $t$ & $b$ & Cost \\
\hline Deb (1991) & 0.2489 & 6.1730 & 8.1789 & 0.2533 & 2.4331 \\
\hline Ragsdell (1976) & 0.2455 & 6.1960 & 8.2730 & 0.2455 & 2.3859 \\
\hline Siddall (1972) & 0.2444 & 6.2189 & 8.2915 & 0.2444 & 2.3815 \\
\hline Coello (1999) & 0.2088 & 3.4205 & 8.9975 & 0.2100 & 1.7483 \\
\hline Present Best & 0.167880 & 4.645894 & 8.928757 & 0.219162 & 1.9000 \\
\hline
\end{tabular}

***Please take note that the results of Welded Beam Design reported in this paper that appears in the Journal and that which appears in Coello(1999) is not correct. 